\title{
THE S-ESTIMATOR IN CHANGE-POINT RANDOM MODEL WITH LONG MEMORY
}

\author{
GABRIELA CIUPERCA \\ Université de Lyon, Université Lyon 1, \\ CNRS, UMR 5208, Institut Camille Jordan, \\ Bat. Braconnier, 43, blvd du 11 novembre 1918, \\ F - 69622 Villeurbanne Cedex, France, \\ email: Gabriela.Ciuperca@univ-lyon1.fr \\ tel: 33(0)4.72.43.16.90, fax: 33(0)4.72.43.16.87
}

\begin{abstract}
The paper considers two-phase random design linear regression models. The errors and the regressors are stationary long-range dependent Gaussian. The regression parameters, the scale parameters and the change-point are estimated using a method introduced by Rousseeuw and Yohai [33]. This is called S-estimator and it has the property that is more robust than the classical estimators; the outliers don't spoil the estimation results. Some asymptotic results, including the strong consistency and the convergence rate of the S-estimators, are proved.
\end{abstract}

Key words: Change-points, S-estimator, Long-memory, Asymptotic properties AMS 2000 subject classifications: primary 62F12; secondary 62H12, 60G15.

\section{Introduction}

Consider the two-phase linear regression model:

$$
Y_{t}=X_{t} \beta_{1} \mathbb{1}_{1 \leq t \leq[n \pi]}+X_{t} \beta_{2} \mathbb{1}_{[n \pi]+1 \leq t \leq n}+\varepsilon_{t}, \quad t=1, \ldots, n
$$

where $\mathbb{1}_{(.)}$is the indicator function and $\pi \in(0,1), \xi=\left(\beta_{1}, \beta_{2}, \pi\right), \beta_{1}, \beta_{2} \in \Upsilon$. The set $\Upsilon$ is a compact of $\mathbb{R}^{d}, d \geq 1$. For this model, $Y_{t}$ denotes the response variable, $X_{t}$ is a p-vector of regressors and $\varepsilon_{t}$ is the error. 
The model parameters are: regression parameters $\beta_{1}$ and $\beta_{2}$, change-point $\pi$ and error variance $\sigma^{2}$, with $\sigma^{2} \in(0, \infty)$. Let us denote $\xi^{0}=\left(\beta_{1}^{0}, \beta_{2}^{0}, \pi^{0}\right)$ and $\sigma_{0}^{2}$ the true values of these parameters. In this paper we consider the problem of estimating of $\xi$ and $\sigma^{2}$, based on the observation of $\left(Y_{t}, X_{t}\right)_{1 \leq t \leq n}$.

Classical estimation methods studied in the statistic literature are the least squares (LS), maximum likelihood (ML) or a wider class M-estimation methods. For each of these methods one has to distinguish the cases when the errors are independent or not, and in the dependent case it is necessary to take into account the covariance structure. The same conditions can be considered for regressors $X_{t}$. In traditional methodology, these variables are usually assumed to be independent or with shortmemory. So, if the errors are i.i.d. or with short-memory, the statistic literature related to the parametric change-point estimation is very vast. Recent developments for the LS estimation include Feder ([13], [14]), Bai and Perron [3], Kim and Kim [22]. Bai [1] considers also the least squares estimation of a shift in linear process. The process $\varepsilon_{t}$ is given by: $\varepsilon_{t}=\sum_{j=0}^{\infty} c_{j} u_{k-j}$, where $u_{j}$ is white noise with mean zero and variance $\sigma^{2}$ and the coefficients $c_{j}$ satisfy $\sum_{j=0}^{\infty} j\left|c_{j}\right|<\infty$. This condition excludes long-memory. For the ML estimation we refer to Bhattacharya [7, Koul and Qian [23], Ciuperca and Dapzol [9]. In the general case of the M-estimator, we can cite the papers of Rukhin and Vajda [34], Koul et al. [25]. Obviously, the list is not exhaustive, the subject is so large and productive that we cannot give all the papers. The convergence rate and limiting distributions of the change-point and of the regression parameters M-estimators are derived for the model (11) by Fiteni [15], under restrictive and numerous assumptions. Among these conditions she considers that $\left(Y_{t}, X_{t}\right)$ is a random vector, $L_{0}-\mathrm{NED}$, on a strong mixing base $\left\{w_{t} ; t=\ldots, 0,1, \ldots\right\}, \rho^{\prime}\left(\varepsilon_{t}+\theta X_{t}\right) X_{t}$ is a random sequence of mean zero, $L_{2}$-NED of size $1 / 2$ on a strong mixing base $\left\{w_{t} ; t=\ldots, 0,1, \ldots\right\}$ and $\sup _{t \leq n} \mathbb{E}\left[\left\|\rho^{\prime}\left(\varepsilon_{t}+\theta X_{t}\right) X_{t}\right\|^{r}\right.$ for some $r>2$. Under the same dependence assumptions, Fiteni [16] considers the $\tau$-estimators.

On the contrary, in the case of long-memory errors or regressors, the statistical literature related to the parametric change-point estimation is less vast. For the simpler model:

$$
Y_{t}=\mu \mathbb{1}_{1 \leq t \leq k^{*}}+(\mu+\delta) \mathbb{1}_{k^{*}<t \leq n}+\varepsilon_{t}
$$

when the errors $\varepsilon_{t}$ are long-memory Gaussian, Horvath and Kokoszka [20] considered the estimator of $k^{*}$ defined by $\hat{k}=\min \left\{k ;\left|U_{k}\right|=\max _{1 \leq i<n}\left|U_{i}\right|\right\}$, where, for $\gamma \in[0,1), U_{i}=\left(\frac{n}{i(n-i)}\right)^{\gamma} \sum_{j=1}^{i}\left(X_{j}-\bar{X}_{n}\right)$. The estimator converges to functionals of fractional Brownian motion. For the same model, Hidalgo and Robinson [19], Sibbertsen [35] consider the LS estimator of $k, \mu$ and $\delta$. A more complex model: $Y_{t}=\mu+\left(\beta+\delta \mathbb{1}_{t<[\tau n]}\right) X_{t}+\varepsilon_{t}$ is considered in the paper of Lazarová [27], but with the supposition that $\tau$ fixed. The limiting distribution of the LS estimator of the parameters $\beta$ and $\delta$ is given. 
Concerning now the estimation method, it is well known that one outlier may cause a large error in a LS-estimator, ML-estimator or more generally in classical M-estimator. Nunes et al. [29], Kuan and Hsu [26] observed that, for the data that have long-memory, the LS-estimator may suggest a spurious change-point when there is none. In that case, the parameters of the model can be estimated by using least absolute deviations (LAD) method. If the errors are independent, Bai [2] studies the LAD estimator for a multiple regime linear regression and Ciuperca [10] for a nonlinear change-point model. A more robust estimator was introduced by Rousseeuw and Yohai [33, by defining the S-estimators as the minimizers of a M-estimator of the residual scale. The interest of the S-estimator in respect to the LAD-estimators, is the breakdown point introduced by Hampel [18]. The breakdown point amounts to determining the smallest contaminating mass that can cause the estimator to take on value arbitrary for from the true value. Instead, concerning this method, to the author's knowledge, the past papers treat only regression models without change-point. For a linear regression model, Davies [11] proves the consistency and weak convergence of S-estimator under the assumption that the errors are i.i.d. random variables. The asymptotic behaviour of the S-estimator in a linear regression, without change-point can be also found in the papers Zhengyan et al. [36], Roeland et al. 32].

In the present paper, we consider a linear regression model with a change-point in an unknown point. The regressors and the errors are assumed to be Gaussian vectors, and respectively variables, with long-memory. The regression parameters, the scale parameter and the change-point location are estimated by the S-method. The difficulty of study of the asymptotic properties of these S-estimators comes especially from the dependence on change-point in the expression of the scale parameter estimators. We first prove that the estimators are strongly convergent and afterwards their convergence rates are obtained. These rates depend of covariance structure of $X_{t}$ and $\varepsilon_{t}$ and of Hermite rank of $\rho\left(\varepsilon_{t} / \sigma_{0}\right)-\mathbb{E}\left[\rho\left(\varepsilon_{t} / \sigma_{0}\right)\right]$, where $\rho$ is the function used to construct the S-estimator. For the regression parameters and the scale parameter, we obtain the same convergence rate as in a model without change-point, let us denote it $v_{n}$. The S-estimator of the change-point has a faster convergence rate, more precisely $n^{-1} v_{n}$. This result is totally different from those obtained in the other papers where the dependence between observations is considered. Especially, let us notice that our change-point S-estimator converges more quickly towards true value than in the independence or the short-memory case or a classic estimation method.

The plan of this paper is as follows. In Section 2 we make some notations and assumptions afterwards we define the S-estimator for a model with change-point. In Section 3, the asymptotic behaviour of these estimator is studied. The proofs of theorems are given in Section 4. Finally, Section 5 contains some lemmas which are useful to prove the main results. 


\section{Notation and assumptions}

Long-memory (long-range dependent) processes arise in numerous physical and social sciences. For several examples, see e.g. Baillie [4], Cheung [8], Lo [28] among others. We also mention Guo and Kuol [17], where some currency exchange data sets with long-memory are considered. Another long-memory example in economy we find also in Ding et al. [12] on S\&P daily 500 stock market returns. In that paper, they found that although the returns themselves contain little serial correlation, the absolute value of returns has significantly positive serial correlation up to 2700 lags.

For the construction of the S-estimators, a function $\rho: \mathbb{R} \longrightarrow[0,1]$ is needed. Throughout our article, we assume that the following classic conditions are satisfied by $\rho$ :

- $\rho$ is symmetric, continuously differentiable on $\mathbb{R}$ and $\rho(0)=0$.

- $\rho$ is increasing in $[0, c)$, for some $c>0$, and constant in $[c, \infty)$.

Let us denote: $\psi(z)=\rho^{\prime}(z)$.

An example of $\rho$ satisfying these conditions was proposed by Beaton and Tukey [5], for some $c>0$ :

$$
\rho(x)=\left\{\begin{array}{cl}
3(x / c)^{2}-3(x / c)^{4}+(x / c)^{6}, & \text { if }|x| \leq c \\
1, & \text { if }|x|>c
\end{array}\right.
$$

For model (11), the following assumptions are considered:

(A1) $X_{t}$ is a sequence of $d$-dimensional stationary long-range dependent Gaussian vectors, with $\mathbb{E}\left[X_{t}\right]=0$, covariance matrix $\Gamma(t)=\mathbb{E}\left[X_{1} X_{t+1}\right]=\mathcal{L}(t)^{T} N(t) \mathcal{L}(t)$, where $N(t)=\operatorname{diag}\left(t^{-\theta_{1}}, \ldots, t^{-\theta_{d}}\right), \theta_{1}, \ldots, \theta_{d} \in(0,1)$ for $t \geq 1$ and $\Gamma(0)=\operatorname{Var}\left(X_{1}\right)$. $\mathcal{L}(x)$ a $d \times d$ orthogonal matrix of slowly varying functions;

(A2) $\varepsilon_{t}$ a sequence of stationary long-range dependent Gaussian variables, with $\mathbb{E}\left[\varepsilon_{t}\right]=0, \gamma(0)=\operatorname{Var}\left[\varepsilon_{t}\right]=\sigma_{0}^{2}$ and the covariance $\gamma(t)=\mathbb{E}\left[\varepsilon_{1} \varepsilon_{t+1}\right]=t^{-\alpha} L(t)$, $\alpha \in(0,1)$ for $t \geq 1$. $L(x)$ a positive slowly varying function;

(A3) the errors $\varepsilon_{t}$ are independent of $X_{t}$.

The values of $\theta_{1}, \ldots, \theta_{d}, \alpha$ and the functions expressions of $\mathcal{L}(x), L(x)$ are known. Recall that a positive measurable function $h$ is slowly varying in Karamata's sense if and only if, for any $\lambda>0, h(\lambda x) / h(x)$ converges to 1 as $x$ tends to infinity. Examples of slowly varying functions: $\log x, \log \log x, \log \log \log x \ldots \ldots$ 
Interested readers are referred to Beran [6] or Robinson [31] for a complete reference on long-memory processes.

An example of process $X_{t}=\left(X_{t 1}, \ldots, X_{t d}\right)$ is obtained when, for some $0<d_{1}<1 / 2$ :

$$
X_{t j}=\sum_{l=1}^{d} \sum_{v \in Z} B_{j l}(t-v) \varsigma_{v, j}, \quad B_{j l}(v)=v^{-\left(1-d_{1}\right)} L_{j l}(v), \quad v \geq 1, \quad j, l=1, \ldots, d
$$

where $L_{j l}$ are slowly varying functions and where $\varsigma_{v}=\left(\varsigma_{v, 1}, \ldots, \varsigma_{v, d}\right)^{T}, v \in Z$ are i.i.d. with $\varsigma_{v, j}, j=1, \ldots, d$ standard Gaussian variables (see Koul and Baillie [24]). For the residual function, let us consider classical notation $r_{t}(\beta)=Y_{t}-X_{t} \beta$ and let $K$ the constant given by $K=\mathbb{E}_{\Phi}\left[\rho\left(\varepsilon_{1} / \sigma_{0}\right)\right]$, where $\Phi$ is standard Gaussian distribution.

In order to construct the S-estimator in a change-point model (1), we proceed as follows:

- first, for $\left(\beta_{1}, \beta_{2}, \pi\right) \in \Upsilon \times \Upsilon \times(0,1)$ fixed, scale parameter $\sigma$ is estimated by the positive solution $s_{n}(\xi)=s_{n}\left(\beta_{1}, \beta_{2}, \pi\right)$ of the equation:

$$
n^{-1} \sum_{t=1}^{[n \pi]} \rho\left(\frac{r_{t}\left(\beta_{1}\right)}{s_{n}(\xi)}\right)+n^{-1} \sum_{t=[n \pi]+1}^{n} \rho\left(\frac{r_{t}\left(\beta_{2}\right)}{s_{n}(\xi)}\right)=K
$$

- at the second stage, the regression parameters are estimated by the argument of the minimum of solution $s_{n}(\xi)$ obtained of the previous phase:

$$
\left(\tilde{\beta}_{1 n}(\pi), \tilde{\beta}_{2 n}(\pi)\right)=\arg \min _{\left(\beta_{1}, \beta_{2}\right) \in \Upsilon \times \Upsilon} s_{n}\left(\beta_{1}, \beta_{2}, \pi\right)
$$

- in the end, the change-point is estimated by:

$$
\hat{\pi}_{n}=\arg \min _{\pi \in[0,1]} s_{n}\left(\tilde{\beta}_{1 n}(\pi), \tilde{\beta}_{2 n}(\pi), \pi\right)
$$

We shall make the usual identifiability assumption that the two segments are different:

$$
\beta_{1} \neq \beta_{2}, \quad \forall \xi \in \Upsilon \times \Upsilon \times(0,1)
$$

i.e. at least one of the coefficients of $X_{t}$ has a shift. Thus the jump at $\pi$ is non-zero. This condition implies that the solution of (66) is unique and it will be essential in the proof of the strong consistency.

If solution $s_{n}(\xi)$ to (44) exists then it is well-defined, bounded, strictly positive, with a probability arbitrarily large (see Lemma 5.1). These results are valid regardless of the covariance structure of $X_{t}$, of $\varepsilon_{t}$ and their distribution. What matters is their average is worth 0 and their variance is bounded.

If (4) has more than one solution, $s_{n}(\xi)$ is defined as the supremum of all solutions. 
Obviously, if function $\rho$ is given by (3) , thus equation (44) has at least a solution.

In this context, we define $\hat{\sigma}_{n}=s_{n}\left(\tilde{\beta}_{1 n}\left(\hat{\pi}_{n}\right), \tilde{\beta}_{2 n}\left(\hat{\pi}_{n}\right), \hat{\pi}_{n}\right)$ as the S-estimator of $\sigma$ and $\left(\hat{\beta}_{1 n}, \hat{\beta}_{2 n}\right)=\left(\tilde{\beta}_{1 n}\left(\hat{\pi}_{n}\right), \tilde{\beta}_{2 n}\left(\hat{\pi}_{n}\right)\right)$ that of $\left(\beta_{1}, \beta_{2}\right)$. We shall study the asymptotic behaviour of $\hat{\sigma}_{n},\left(\hat{\beta}_{1 n}, \hat{\beta}_{2 n}\right)$ and of $\hat{\pi}_{n}$, in the case that equation (4) has at least a solution.

For any $\varphi$ twice differentiable function, for $x, h \in \mathbb{R}$, throughout this paper we are going to use the mean value theorem under the form:

$$
\varphi(x+h)=\varphi(x)+h\left[\varphi^{\prime}(x)+h \int_{0}^{1}(1-s) \varphi^{\prime \prime}(x+s h) d s\right]
$$

For a vector $V=\left(v_{1}, \cdots, v_{m}\right)$, let us denote by $\|V\|$ its Euclidean norm and we make the convention that $|V|=\left(\left|v_{1}\right|, \cdots,\left|v_{m}\right|\right)$.

In the following, we denote by $C$ a generic positive finite constant that may be different in different context, but will never depend on $n$.

\section{Asymptotic behaviour}

This section establishes asymptotic properties of the S-estimator in model (1). For this purpose, first let us calculate, for solution $s_{n}(\xi)$ of equation (4), the partial derivatives with respect to $\beta_{1}$ and $\beta_{2}$. Differentiating (41) with respect to $\beta_{1}$, we obtain:

$\sum_{t=1}^{[n \pi]} \frac{r_{t}\left(\beta_{1}\right)}{s_{n}(\xi)} \frac{\partial s_{n}(\xi)}{\partial \beta_{1}} \psi\left(\frac{r_{t}\left(\beta_{1}\right)}{s_{n}(\xi)}\right)+\sum_{t=[n \pi]+1}^{n} \frac{r_{t}\left(\beta_{2}\right)}{s_{n}(\xi)} \frac{\partial s_{n}(\xi)}{\partial \beta_{1}} \psi\left(\frac{r_{t}\left(\beta_{2}\right)}{s_{n}(\xi)}\right)+\sum_{t=1}^{[n \pi]} \frac{X_{t}}{s_{n}(\xi)} \psi\left(\frac{r_{t}\left(\beta_{1}\right)}{s_{n}(\xi)}\right)=0$

Considering the following notation:

$$
D_{n}(\xi)=n^{-1} \sum_{t=1}^{[n \pi]} \frac{r_{t}\left(\beta_{1}\right)}{s_{n}(\xi)} \psi\left(\frac{r_{t}\left(\beta_{1}\right)}{s_{n}(\xi)}\right)+n^{-1} \sum_{t=[n \pi]+1}^{n} \frac{r_{t}\left(\beta_{2}\right)}{s_{n}(\xi)} \psi\left(\frac{r_{t}\left(\beta_{2}\right)}{s_{n}(\xi)}\right)
$$

and by making similar calculation for $\partial s_{n}(\xi) / \partial \beta_{2}$, we obtain:

$$
\left\{\begin{array}{l}
\frac{\partial s_{n}(\xi)}{\partial \beta_{1}}=-n^{-1} D_{n}(\xi)^{-1} \sum_{t=1}^{[n \pi]} \frac{X_{t}}{s_{n}(\xi)} \psi\left(\frac{r_{t}\left(\beta_{1}\right)}{s_{n}(\xi)}\right) \\
\frac{\partial s_{n}(\xi)}{\partial \beta_{2}}=-n^{-1} D_{n}(\xi)^{-1} \sum_{t=[n \pi]+1}^{n} \frac{X_{t}}{s_{n}(\xi)} \psi\left(\frac{r_{t}\left(\beta_{2}\right)}{s_{n}(\xi)}\right)
\end{array}\right.
$$


Since $\rho$ is symmetric and increasing in $[0, c)$ ( and choosing suitably $c$ ) we have:

$$
x \psi(x)\left\{\begin{array}{l}
>0, \text { if } x \in(-c, c) \backslash\{0\} \\
=0, \text { if } x=0 \text { or }|x| \geq c
\end{array}\right.
$$

By means of Lemma 5.2, we prove that the random process $D_{n}(\xi)^{-1}$ is bounded with a probability close to 1 . In fact, the covariance structure of $X_{t}$ and of $\varepsilon_{t}$, respectively, plays no role in this result. Moreover, if both random variables are no more Gaussian, Lemma 5.2 holds if $X_{t}$ and $\varepsilon_{t}$ are bounded with a probability close to 1 .

In order to prove the consistency we require that function $\psi$ also is differentiable and strictly increasing on $(0, c)$. This condition will be used for the Taylor's expansion of $\rho$, around $\left(\beta_{1}^{0}, \beta_{2}^{0}\right)$, up to second order.

(H1) $\psi($.$) is differentiable and \psi^{\prime}(u)>0, \forall u \in(0, c)$.

Theorem 3.1 Under assumptions (A1)-(A3), (H1), (7), we have that estimator $\hat{\xi}_{n}=\left(\hat{\beta}_{1 n}, \hat{\beta}_{2 n}, \hat{\pi}_{n}\right)$ is strongly consistent: $\hat{\xi}_{n} \underset{n \rightarrow \infty}{\stackrel{a . s .}{\rightarrow}} \xi_{0}$.

Remark 3.1 Statement of Theorem 3.1 remains valid, if $X_{t}$ is not Gaussian, but it is i.i.d. and $\mathbb{E}\left[X_{t} X_{t}^{T}\right]<\infty$. If $\varepsilon_{t}$ is not Gaussian, it has to be bounded with a probability close to 1 .

As a consequence of relation (10), the first two stages (40) and (5) in the construction of the parameters estimators, are the solutions to the equations system:

$$
\begin{aligned}
& \text { (a) } n^{-1} \sum_{t=1}^{[n \pi]} \rho\left(\frac{r_{t}\left(\beta_{1}\right)}{\sigma}\right)+n^{-1} \sum_{t=[n \pi]+1}^{n} \rho\left(\frac{r_{t}\left(\beta_{2}\right)}{\sigma}\right)-K=0 \\
& \text { (b) } n^{-1} \sum_{t=1}^{[n \pi]} \psi\left(\frac{r_{t}\left(\beta_{1}\right)}{\sigma}\right) X_{t}=0 \\
& \text { (c) } n^{-1} \sum_{t=[n \pi]+1}^{n} \psi\left(\frac{r_{t}\left(\beta_{2}\right)}{\sigma}\right) X_{t}=0
\end{aligned}
$$

Since the change-point intervention is essential, the convergence study of the scale parameter estimator is realized separately. According to Theorem [3.1, we fix $\pi$ in a neighbourhood $\mathcal{V}\left(\pi^{0}\right)$ of $\pi^{0}$. In order to show the convergence of the scale parameter estimator, supplementary assumptions are needed.

(H2) $\psi$ is twice differentiable with bounded second derivative.

(H3) $\psi(x) / x$ is nonincreasing for $x>0$. 
Obviously, function (3) satisfies assumptions (H1)-(H3). As will be seen below, assumption (H2) is needed to control the rest in the Taylor's expansion of $s_{n}(\xi)$, while (H3) is used in order to apply results of Zhengyan et al. [36] on the consistency of the scale S-estimator in a model without change-point. Moreover, in the paper of Zhengyan et al. [36], the assumption (H3) is needed to show the convergence of the regression parameter estimator, which is not the case here.

Theorem 3.2 Under (A1)-(A3), (H1)-(H3), (7), for all $\pi$ in a neighbourhood $\mathcal{V}\left(\pi^{0}\right)$ of $\pi^{0}$, the estimator of $\sigma_{0}$ is strongly consistent: $s_{n}\left(\tilde{\beta}_{1 n}(\pi), \tilde{\beta}_{2 n}(\pi), \pi\right) \underset{n \rightarrow \infty}{\stackrel{\text { a.s. }}{\rightarrow}}$ $\sigma_{0}$.

Corollary 3.1 Under (A1)-(A3), (H1)-(H3), (7), scale parameter S-estimator $\hat{\sigma}_{n}=s_{n}\left(\hat{\beta}_{1 n}, \hat{\beta}_{2 n}, \hat{\pi}_{n}\right)$ is strongly consistent for $\sigma_{0}$.

Remark 3.2 In a model without change-point, the assumption (H2) is needed for found the convergence rate and the asymptotic distribution of the estimators but not in the convergence proof.

Remark 3.3 The convergence result of Theorem 3.2 holds if random vector $X_{t}$ is not more Gaussian but i.i.d. with $\mathbb{E}\left[X_{t}\right]=0$ and $\mathbb{E}\left[X_{t} X_{t}^{T}\right]<\infty$.

In order to find the convergence rate, we will use the Hermite expansion for a function of standard Gaussian variable (for details about the Hermite expansion see for example Palma [30]). Let us consider function $\chi():.=\rho()-$.$K , where$ $K=\mathbb{E}_{\Phi}\left[\rho\left(\varepsilon_{1} / \sigma_{0}\right)\right]$. Suppose that the Hermite rank of $\chi\left(\frac{\varepsilon_{1}}{\sigma_{0}}\right)$ is $q_{1}$. Because function $\rho$ is symmetric and $\rho(0)=0$, we have $q_{1} \geq 2$. If we denote $\nu_{t}=\varepsilon_{t} / \sigma_{0}$, then:

$$
\chi\left(\nu_{t}\right)=\sum_{q \geq q_{1}} \frac{J_{q}(\chi)}{q !} H_{q}\left(\nu_{t}\right)
$$

with $H_{q}$ the Hermite polynomial, $J_{q}(\chi)=\mathbb{E}\left[\chi\left(\nu_{1}\right) H_{q}\left(\nu_{1}\right)\right]$ and for all $t, t^{\prime}=1, \cdots n$ :

$$
\mathbb{E}\left[H_{p}\left(\nu_{t}\right) H_{q}\left(\nu_{t^{\prime}}\right)\right]=q ! \gamma^{q}\left(t-t^{\prime}\right) \mathbb{1}_{p=q}
$$

Let also $k=\min \left\{\left(\alpha q_{1}\right) / 2,\left(\theta_{i}+\alpha\right) / 2,1 \leq i \leq d\right\}$.

In order to have the rate of convergence of the estimators in a model without change-point, following assumptions are imposed by Zhengyan et al [36]: $\alpha q_{1}<1$ and $\max \left\{\alpha+\theta_{j} ; 1 \leq j \leq d\right\}$.

The following theorem gives the convergence rate of the regression parameters and of the scale parameter estimators. These rates are the same that in a model with- 
out change-point.

Theorem 3.3 For all $\pi \in(0,1)$, if (A1)-(A3), (H1)-(H3), (7) hold, we have

$$
\begin{gathered}
\left\|\tilde{\beta}_{1 n}(\pi)-\beta_{1}^{0}\right\|=O_{\mathbb{P}}\left((n \pi)^{-k} L_{1}(n \pi)\right)=O_{\mathbb{P}}\left(n^{-k} \tilde{L}_{1}(n)\right) \\
\left\|\tilde{\beta}_{2 n}(\pi)-\beta_{2}^{0}\right\|=O_{\mathbb{P}}\left((n(1-\pi))^{-k} L_{1}(n(1-\pi))\right)=O_{\mathbb{P}}\left(n^{-k} \tilde{L}_{1}(n)\right)
\end{gathered}
$$

where $L_{1}$ and $\tilde{L}_{1}$ are slowly varying functions. For the scale parameter, putting $\tilde{s}_{n}(\pi):=s_{n}\left(\tilde{\beta}_{1 n}(\pi), \tilde{\beta}_{2 n}(\pi), \pi\right)$, we have $\left|\tilde{s}_{n}(\pi)-\sigma^{0}\right|=O_{\mathbb{P}}\left(n^{-k} \tilde{L}_{1}(n)\right)$.

Now let us study the convergence rate of the change-point estimator:

$\hat{\pi}_{n}=\arg \min _{\pi} s_{n}\left(\tilde{\beta}_{1 n}(\pi), \tilde{\beta}_{2 n}(\pi), \pi\right)=\arg \min _{\pi}\left[s_{n}\left(\tilde{\beta}_{1 n}(\pi), \tilde{\beta}_{2 n}(\pi), \pi\right)-s_{n}\left(\beta_{1}^{0}, \beta_{2}^{0}, \pi\right)\right]$

For that we consider one of the last two equations of (12), for instance (c):

$$
n^{-1} \sum_{t=[n \pi]+1}^{n} \psi\left(\frac{r_{t}\left(\tilde{\beta}_{2 n}(\pi)\right)}{s_{n}\left(\tilde{\beta}_{1 n}(\pi), \tilde{\beta}_{2 n}(\pi), \pi\right)}\right) X_{t}=0
$$

Theorem 3.4 Under assumptions (A1)-(A3), (H1)-(H3), (7), we have $\hat{\pi}_{n}-\pi^{0}=$ $O_{\mathbb{P}}\left(n^{-1-k} \tilde{L}_{1}(n)\right)$ with $\tilde{L}_{1}(n)$ a slowly varying function.

Example. If $\alpha \geq \max _{i=1, \ldots, d} \theta_{i}$, then $k=\left(\alpha+\min _{i=1, \ldots, d} \theta_{i}\right) / 2 \leq \alpha$.

What is remarkable comparatively to the independence or the short-memory case is that $\hat{\pi}_{n}$ converges faster towards $\pi_{0}$ when $X_{t}$ or $\varepsilon_{t}$ are long-range dependent. Consider the particular case $\alpha=\theta_{1}=\ldots=\theta_{d}$, then $k=\alpha$. Further if $\alpha \in(1 / 2,1)$, then, for the estimators of $\beta_{1}$ and $\beta_{2}$, we have a faster convergence rate than in the independence or short-memory case. Finally, the long-memory brings about that the true values of the parameters are faster approached.

Remark also that the obtained convergence rate completely differs from that of change-point $\tau$-estimators when $X_{t}$ are NED-dependent (Fiteni [15], [16]). If $X_{t}$ are independent, the convergence rate is $n^{-1}$ for the change-point estimator and $n^{-1 / 2}$ for the parameters regression estimator, indifferently of used method: M-method (Koul et al. [25]), ML-method (Ciuperca and Dapzol [9]), LS-estimation (Bai and Perron [3]). Same convergence rate, $n^{-1}$, is obtained for change-point LS-estimator in a model with correlated errors, but not with long-memory (Bai [1]). 
It is interesting to note that the rate convergence of the change-point estimator in the mean of Gaussian variable (2), having long-range dependence, considered by Horvath and Kokoszka [20], is $n^{-1} g^{-1}(1 / \delta)$ with $g$ a regular varying function. Thus, the estimator of Horvath and Kokoszka [20] is slower than our estimator. On the other hand, let us remark that convergence rate of the S-estimators depends of the Hermite rank of $\rho\left(\varepsilon_{1} / \sigma_{0}\right)-K$ and of the covariance structure of $X_{t}$ and $\varepsilon_{t}$.

\section{Proofs of Theorems}

Proof of Theorem 3.1. Let us consider the function $e(\xi)=\mathbb{E}\left[s_{n}(\eta, \pi)-s_{n}\left(\eta^{0}, \pi^{0}\right)\right]$, with supposition, without loss the generality, that $\pi \leq \pi^{0}$. Using the same arguments as for (37), we obtain that: $\mathbb{E}\left[\left|s_{n}(\eta, \pi)-s_{n}\left(\eta, \pi^{0}\right)\right|\right] \leq C\left\|\beta_{1}-\beta_{2}\right\| \cdot\left|\pi-\pi^{0}\right|<$ $\infty$ and similarly to (34): $\mathbb{E}\left[\left|s_{n}\left(\eta, \pi^{0}\right)-s_{n}\left(\eta^{0}, \pi^{0}\right)\right|\right] \leq C\left\|\eta-\eta^{0}\right\|$. Thus, function $e(\xi)$ is well-defined. By Lemma 5.3, function $e(\xi)$ is continuous and furthermore $e\left(\xi^{0}\right)=0$. For using an argument like the one in Huber [21], we will to prove that: $\mathbb{E}\left[s_{n}(\eta, \pi)-s_{n}\left(\eta^{0}, \pi^{0}\right)\right]>0$, for every $\xi \neq \xi^{0}$. Since $s_{n}(\xi)$ and $s_{n}\left(\xi^{0}\right)$ are both solutions of equation (4), we have $0=\left(S_{1, n}^{(0)}+S_{1, n}^{(1)}\right)+\left(S_{2, n}^{(0)}+S_{2, n}^{(1)}\right)+\left(S_{3, n}^{(0)}+S_{3, n}^{(1)}\right)$, with:

$$
\begin{aligned}
S_{1, n}^{(0)} & \equiv n^{-1} \sum_{t=1}^{[n \pi]}\left[\rho\left(\frac{r_{t}\left(\beta_{1}^{0}\right)}{s_{n}(\xi)}\right)-\rho\left(\frac{r_{t}\left(\beta_{1}^{0}\right)}{s_{n}\left(\xi^{0}\right)}\right)\right], S_{1, n}^{(1)} \equiv n^{-1} \sum_{t=1}^{[n \pi]}\left[\rho\left(\frac{r_{t}\left(\beta_{1}\right)}{s_{n}(\xi)}\right)-\rho\left(\frac{r_{t}\left(\beta_{1}^{0}\right)}{s_{n}(\xi)}\right)\right] \\
S_{2, n}^{(0)} & \equiv n^{-1} \sum_{t=[n \pi]+1}^{\left[n \pi^{0}\right]}\left[\rho\left(\frac{r_{t}\left(\beta_{1}^{0}\right)}{s_{n}(\xi)}\right)-\rho\left(\frac{r_{t}\left(\beta_{1}^{0}\right)}{s_{n}\left(\xi^{0}\right)}\right)\right], S_{2, n}^{(1)} \equiv n^{-1} \sum_{t=[n \pi]+1}^{\left[n \pi^{0}\right]}\left[\rho\left(\frac{r_{t}\left(\beta_{2}\right)}{s_{n}(\xi)}\right)-\rho\left(\frac{r_{t}\left(\beta_{1}^{0}\right)}{s_{n}(\xi)}\right)\right] \\
S_{3, n}^{(0)} & \equiv n^{-1} \sum_{t=\left[n \pi^{0}\right]+1}^{n}\left[\rho\left(\frac{r_{t}\left(\beta_{2}^{0}\right)}{s_{n}(\xi)}\right)-\rho\left(\frac{r_{t}\left(\beta_{2}^{0}\right)}{s_{n}\left(\xi^{0}\right)}\right)\right], S_{3, n}^{(1)} \equiv n^{-1} \sum_{t=\left[n \pi^{0}\right]+1}^{n}\left[\rho\left(\frac{r_{t}\left(\beta_{2}\right)}{s_{n}(\xi)}\right)-\rho\left(\frac{r_{t}\left(\beta_{2}^{0}\right)}{s_{n}(\xi)}\right)\right]
\end{aligned}
$$

Then, by the mean value theorem (TVM), $S_{1, n}^{(0)}+S_{2, n}^{(0)}+S_{3, n}^{(0)}$ can be written as:

$$
\begin{aligned}
n^{-1}\left(\frac{1}{s_{n}(\xi)}-\frac{1}{s_{n}\left(\xi^{0}\right)}\right) & {\left[\sum_{t=1}^{[n \pi]} r_{t}\left(\beta_{1}^{0}\right) \psi\left(\frac{r_{t}\left(\beta_{1}^{0}\right)}{u_{n}^{(1)}\left(\eta^{0}, \pi, \pi^{0}\right)}\right)+\sum_{t=[n \pi]+1}^{\left[n \pi^{0}\right]} r_{t}\left(\beta_{1}^{0}\right) \psi\left(\frac{r_{t}\left(\beta_{1}^{0}\right)}{u_{n}^{(2)}\left(\eta^{0}, \pi, \pi^{0}\right)}\right)\right.} \\
& \left.+\sum_{t=\left[n \pi^{0}\right]+1}^{n} r_{t}\left(\beta_{2}^{0}\right) \psi\left(\frac{r_{t}\left(\beta_{2}^{0}\right)}{u_{n}^{(3)}\left(\eta^{0}, \pi, \pi^{0}\right)}\right)\right]
\end{aligned}
$$

with $u_{n}^{(1)}, u_{n}^{(2)}, u_{n}^{(3)}$ defined in the same way as in the proof of the Lemma 5.3 , Moreover, using property (11), we have the following: $S_{1, n}^{(0)}+S_{2, n}^{(0)}+S_{3, n}^{(0)}=\left[s_{n}\left(\xi^{0}\right)-\right.$ $\left.s_{n}(\xi)\right] V_{n}$, where $V_{n}$ is a positive random variable with probability close to 1 . 
Moreover, using Taylor's expansion, the expressions of $S_{1, n}^{(1)}, S_{2, n}^{(1)}$ and $S_{3, n}^{(1)}$ can be written as:

$$
\begin{aligned}
& S_{1, n}^{(1)}=n^{-1} \sum_{t=1}^{[n \pi]} X_{t}\left(\beta_{1}^{0}-\beta_{1}\right)\left[\psi\left(\frac{r_{t}\left(\beta_{1}^{0}\right)}{s_{n}(\xi)}\right)+\frac{1}{2} \psi^{\prime}\left(\frac{\varepsilon_{t}+\delta_{1} X_{t}\left(\beta_{1}^{0}-\beta_{1}\right)}{s_{n}(\xi)}\right)\left(\beta_{1}^{0}-\beta_{1}\right)^{T} X_{t}^{T}\right] \\
& S_{2, n}^{(1)}=n^{-1} \sum_{t=[n \pi]+1}^{\left[n \pi^{0}\right]} X_{t}\left(\beta_{1}^{0}-\beta_{2}\right)\left[\psi\left(\frac{r_{t}\left(\beta_{1}^{0}\right)}{s_{n}(\xi)}\right)+\frac{1}{2} \psi^{\prime}\left(\frac{\varepsilon_{t}+\delta_{2} X_{t}\left(\beta_{1}^{0}-\beta_{2}\right)}{s_{n}(\xi)}\right)\left(\beta_{1}^{0}-\beta_{2}\right)^{T} X_{t}^{T}\right] \\
& S_{3, n}^{(1)}=n^{-1} \sum_{t=\left[n \pi^{0}\right]+1}^{n} X_{t}\left(\beta_{2}^{0}-\beta_{2}\right)\left[\psi\left(\frac{r_{t}\left(\beta_{2}^{0}\right)}{s_{n}(\xi)}\right)+\frac{1}{2} \psi^{\prime}\left(\frac{\varepsilon_{t}+\delta_{3} X_{t}\left(\beta_{2}^{0}-\beta_{2}\right)}{s_{n}(\xi)}\right)\left(\beta_{2}^{0}-\beta_{2}\right)^{T} X_{t}^{T}\right]
\end{aligned}
$$

with $\delta_{1}, \delta_{2}, \delta_{3} \in(0,1)$. By the ergodic theorem, we obtain:

$$
\begin{aligned}
& n^{-1} \sum_{t=1}^{[n \pi]} X_{t}\left(\beta_{1}^{0}-\beta_{1}\right) \psi\left(\frac{r_{t}\left(\beta_{1}^{0}\right)}{s_{n}(\xi)}\right)=o_{\mathbb{P}}(1), n^{-1} \sum_{t=[n \pi]+1}^{\left[n \pi^{0}\right]} X_{t}\left(\beta_{1}^{0}-\beta_{2}\right) \psi\left(\frac{r_{t}\left(\beta_{1}^{0}\right)}{s_{n}(\xi)}\right)=o_{\mathbb{P}}(1), \\
& n^{-1} \sum_{t=\left[n \pi^{0}\right]+1}^{n} X_{t}\left(\beta_{2}^{0}-\beta_{2}\right) \psi\left(\frac{r_{t}\left(\beta_{2}^{0}\right)}{s_{n}(\xi)}\right)=o_{\mathbb{P}}(1)
\end{aligned}
$$

Relation (14) and assumption (H1) imply: for any $\xi \neq \xi^{0}$, for all $\epsilon>0$, there exits $a>0$, such that

$$
\mathbb{P}\left[S_{1, n}^{(1)}+S_{2, n}^{(1)}+S_{3, n}^{(1)}>a\right]>1-\epsilon
$$

Assumption (7), the above relation and $S_{1, n}^{(1)}+S_{2, n}^{(1)}+S_{3, n}^{(1)}=-\left(S_{1, n}^{(0)}+S_{2, n}^{(0)}+S_{3, n}^{(0)}\right)=$ $\left[s_{n}(\xi)-s_{n}\left(\xi^{0}\right)\right] V_{n}$, with $V_{n}>0$, imply the conclusion $\mathbb{E}\left[s_{n}(\eta, \pi)-s_{n}\left(\eta^{0}, \pi^{0}\right)\right]>$ 0 , for all $\xi \neq \xi^{0}$. Using this, the compactness of the parameter space, $\hat{\xi}_{n}=$ $\arg \min _{\xi \in \Upsilon \times \Upsilon \times[0,1]} s_{n}(\xi)$ and an argument like one in Huber [21], the strongly convergence of $\hat{\xi}_{n}$ results.

Proof of Theorem 3.2. We first prove that, if we consider in (11) the true value for $\eta$ and $\pi$, then the scale parameter estimator is strongly consistent:

$$
s_{n}\left(\eta^{0}, \pi^{0}\right) \underset{n \rightarrow \infty}{\stackrel{a . s .}{\longrightarrow}} \sigma_{0}
$$

Let us observe that in fact $s_{n}\left(\eta^{0}, \pi^{0}\right)$ is the solution of a problem without breaking:

$$
K=n^{-1} \sum_{t=1}^{\left[n \pi^{0}\right]} \rho\left(\frac{\varepsilon_{t}}{s_{n}\left(\xi^{0}\right)}\right)+n^{-1} \sum_{t=\left[n \pi^{0}\right]+1}^{n} \rho\left(\frac{\varepsilon_{t}}{s_{n}\left(\xi^{0}\right)}\right)=n^{-1} \sum_{t=1}^{n} \rho\left(\frac{\varepsilon_{t}}{s_{n}\left(\xi^{0}\right)}\right)
$$

and then, relation (16) is obtained by Theorem 3.1 of Zhengyan et al. [36]. Now, as a consequence of Theorem 3.1 , we may consider only the case $(\eta, \pi)$ in a neigh- 
bourhood $\mathcal{V}\left(\eta^{0}, \pi^{0}\right)$ of $\left(\eta^{0}, \pi^{0}\right)$. Consider the decomposition:

$s_{n}(\eta, \pi)-s_{n}\left(\eta^{0}, \pi^{0}\right)=\left[s_{n}(\eta, \pi)-s_{n}\left(\eta^{0}, \pi\right)\right]+\left[s_{n}\left(\eta^{0}, \pi\right)-s_{n}\left(\eta^{0}, \pi^{0}\right)\right]: \equiv S_{1}(n)+S_{2}(n)$

Since $S_{1}(n)$, depends only on the regression parameters, by Theorem 3.1, taking into account relations (10) and (32), we readily obtain:

$$
\sup _{\eta \in \mathcal{V}\left(\eta^{0}\right)}\left|s_{n}(\eta, \pi)-s_{n}\left(\eta^{0}, \pi\right)\right| \underset{n \rightarrow \infty}{\stackrel{a . s .}{\longrightarrow}} 0
$$

For $S_{2}(n)$, an argument like the one used for (35) yield that $s_{n}\left(\eta^{0}, \pi\right)-s_{n}\left(\eta^{0}, \pi^{0}\right)$ behaves as:

$$
n^{-1} \sum_{t=[n \pi]+1}^{\left[n \pi^{0}\right]} X_{t} \psi\left(\frac{\tilde{r}_{t}\left(\beta_{1}^{0}, \beta_{2}^{0}\right)}{s_{n}\left(\eta^{0}, \pi\right)}\right)
$$

where $\tilde{r}_{t}\left(\beta_{1}^{0}, \beta_{2}^{0}\right)=r_{t}\left(\beta_{1}^{0}\right)+m_{t}\left[r_{t}\left(\beta_{2}^{0}\right)-r_{t}\left(\beta_{1}^{0}\right)\right]$, with $0<m_{t}<1$. Let us remark that $\tilde{r}_{t}\left(\beta_{1}^{0}, \beta_{2}^{0}\right)=\varepsilon_{t}$. We write Taylor's expansion of $\psi\left(\varepsilon_{t} / s_{n}\left(\eta^{0}, \pi\right)\right)$ around $\psi\left(\varepsilon_{t} / \sigma_{0}\right)$ up to second order:

$$
\begin{gathered}
n^{-1} \sum_{t=1}^{\left[n \pi^{0}\right]} X_{t} \psi\left(\frac{\varepsilon_{t}}{s_{n}\left(\eta^{0}, \pi\right)}\right)=n^{-1} \sum_{t=1}^{\left[n \pi^{0}\right]} X_{t} \psi\left(\frac{\varepsilon_{t}}{\sigma_{0}}\right)-n^{-1} \frac{s_{n}\left(\eta^{0}, \pi\right)-\sigma_{0}}{\sigma_{0} s_{n}\left(\eta^{0}, \pi\right)} \sum_{t=1}^{\left[n \pi^{0}\right]} X_{t} \varepsilon_{t} \psi^{\prime}\left(\frac{\varepsilon_{t}}{\sigma_{0}}\right) \\
+n^{-1} \frac{\left(s_{n}\left(\eta^{0}, \pi\right)-\sigma_{0}\right)^{2}}{2 \sigma_{0} s_{n}\left(\eta^{0}, \pi\right)} \sum_{t=1}^{\left[n \pi^{0}\right]} \psi^{\prime \prime}\left(\varsigma_{t}\right) \varepsilon_{t}^{2} X_{t}
\end{gathered}
$$

with $\varsigma_{t}=\varepsilon_{t}\left[s_{n}\left(\eta^{0}, \pi\right)+v_{t}\left(\sigma_{0}-s_{n}\left(\eta^{0}, \pi\right)\right)\right] /\left(\sigma_{0} s_{n}\left(\eta^{0}, \pi\right)\right), v_{t} \in(0,1)$. Since $\psi^{\prime \prime}$ is bounded, we have $n^{-1} \sum_{t=1}^{\left[n \pi^{0}\right]} \psi^{\prime \prime}\left(\varsigma_{t}\right) \varepsilon_{t}^{2} X_{t}<\infty$ with probability 1. Moreover:

$$
\pi^{0}\left[\frac{1}{\left[n \pi^{0}\right]} \sum_{t=1}^{\left[n \pi^{0}\right]} X_{t} \varepsilon_{t} \psi^{\prime}\left(\frac{\varepsilon_{t}}{\sigma_{0}}\right)\right] \underset{n \rightarrow \infty}{\stackrel{a . s .}{\longrightarrow}} \pi^{0} \mathbb{E}\left[X_{t} \varepsilon_{t} \psi^{\prime}\left(\frac{\varepsilon_{t}}{\sigma_{0}}\right)\right]=0
$$

Hence: $n^{-1} \sum_{t=1}^{\left[n \pi^{0}\right]} X_{t}\left[\psi\left(\varepsilon_{t} / s_{n}\left(\eta^{0}, \pi\right)\right)-\psi\left(\varepsilon_{t} / \sigma_{0}\right)\right]=o_{\mathbb{P}}\left(s_{n}\left(\eta^{0}, \pi\right)-\sigma_{0}\right)$. This relation and $n^{-1} \sum_{t=1}^{\left[n \pi^{0}\right]} X_{t} \psi\left(\varepsilon_{t} / \sigma_{0}\right) \underset{n \rightarrow \infty}{\stackrel{a . s}{\longrightarrow}} 0$ yield that $S_{2}(n)=s_{n}\left(\eta^{0}, \pi\right)-s_{n}\left(\eta^{0}, \pi^{0}\right)=$ $o_{\mathbb{P}}(1)+o_{\mathbb{P}}\left(s_{n}\left(\eta^{0}, \pi\right)-\sigma_{0}\right)=o_{\mathbb{P}}(1)+o_{\mathbb{P}}\left(S_{2}(n)\right)$, for the last relation we have used (16). Then $\sup _{\pi \in \mathcal{V}\left(\pi^{0}\right)}\left|S_{2}(n)\right| \underset{n \rightarrow \infty}{\stackrel{a . s}{\rightarrow}} 0$. This fact, with relation (18), together with decomposition (17) and relation (16), yield the Theorem.

Proof of Theorem 3.3. For $\pi \in(0,1)$ fixed, the convergence rate of the regression parameters estimator $\tilde{\beta}_{1 n}(\pi)$ and $\tilde{\beta}_{2 n}(\pi)$ is obtained by the application of Zhengyan et al. [36] results on every segment. On the other hand, the study of the convergence rate of $\hat{s}_{n}$ is more difficult because it interferes in both segments. For notational 
simplicity, in the rest of this proof, we denote $\tilde{\beta}_{1 n}=\tilde{\beta}_{1 n}(\pi), \tilde{\beta}_{2 n}=\tilde{\beta}_{2 n}(\pi)$ and $\tilde{s}_{n}=\tilde{s}_{n}(\pi)$. The study will be made in two stages. First, we are going to write equation (12) (a) in another form, putting in evidence $\sigma_{0}$ by a limited development. Afterwards, in the second stage, the obtained form is studied by taking into account the convergence rate of the regression parameters estimators and what $X_{t}, \varepsilon_{t}$ are long-memory Gaussian.

Stage 1. Equation (12) (a) can be expressed as:

$$
n^{-1} \sum_{t=1}^{[n \pi]} \chi\left(\frac{r_{t}\left(\tilde{\beta}_{1 n}\right)}{\tilde{s}_{n}}\right)+n^{-1} \sum_{t=[n \pi]+1}^{n} \chi\left(\frac{r_{t}\left(\tilde{\beta}_{2 n}\right)}{\tilde{s}_{n}}\right)=0
$$

We apply (8) to function $\chi$ and for:

$t=1, \cdots,[n \pi], x_{t}=r_{t}\left(\tilde{\beta}_{1 n}\right) / \tilde{s}_{n}, h_{t}=\left(\sigma_{0}^{-1}-\tilde{s}_{n}^{-1}\right) r_{t}\left(\tilde{\beta}_{1 n}\right)$
$t=[n \pi]+1, \cdots, n, x_{t}=r_{t}\left(\tilde{\beta}_{2 n}\right) / \tilde{s}_{n}, h_{t}=\left(\sigma_{0}^{-1}-\tilde{s}_{n}^{-1}\right) r_{t}\left(\tilde{\beta}_{2 n}\right)$

Hence, for the part $t=1, \cdots,[n \pi]$, we have:

$$
\begin{aligned}
n^{-1} \sum_{t=1}^{[n \pi]} \chi\left(x_{t}+h_{t}\right)= & n^{-1} \sum_{t=1}^{[n \pi]} \chi\left(x_{t}\right)+\frac{\tilde{s}_{n}-\sigma_{0}}{\sigma_{0} \tilde{s}_{n}}\left[n^{-1} \sum_{t=1}^{[n \pi]} r_{t}\left(\tilde{\beta}_{1 n}\right) \psi\left(x_{t}\right)\right. \\
& \left.+n^{-1} \frac{\tilde{s}_{n}-\sigma_{0}}{\sigma_{0} \tilde{s}_{n}} \sum_{t=1}^{[n \pi]} r_{t}^{2}\left(\tilde{\beta}_{1 n}\right) \int_{0}^{1}(1-s) \psi^{\prime}\left(\frac{r_{t}\left(\tilde{\beta}_{1 n}\right)}{\sigma_{0}}+s \cdot r_{t}\left(\tilde{\beta}_{1 n}\right)\left(\frac{1}{\sigma_{0}}-\frac{1}{\tilde{s}_{n}}\right)\right) d s\right]
\end{aligned}
$$

Thus, in order to study the first sum of (19), we shall analyse the terms of the right-hand side of (20).

We first consider the last term of the right-hand side of (20). Elementary algebra yields that:

$$
\begin{array}{r}
n^{-1} \frac{\tilde{s}_{n}-\sigma_{0}}{\sigma_{0} \tilde{s}_{n}} \sum_{t=1}^{[n \pi]} r_{t}^{2}\left(\tilde{\beta}_{1 n}\right)=\frac{\tilde{s}_{n}-\sigma_{0}}{\sigma_{0} \tilde{s}_{n}}\left[n^{-1} \sum_{t=1}^{[n \pi]} \varepsilon_{t}^{2}+n^{-1} \sum_{t=1}^{[n \pi]}\left(\tilde{\beta}_{1 n}-\beta_{1}^{0}\right)^{T} X^{T} X_{t}\left(\tilde{\beta}_{1 n}-\beta_{1}^{0}\right)\right. \\
\left.+2 n^{-1} \sum_{t=1}^{[n \pi]} \varepsilon_{t} X_{t}\left(\tilde{\beta}_{1 n}-\beta_{1}^{0}\right)\right]
\end{array}
$$

By the ergodic theorem $n^{-1} \sum_{t=1}^{[n \pi]} \varepsilon_{t}^{2}=O_{\mathbb{P}}(1), n^{-1} \sum_{t=1}^{[n \pi]} X^{T} X_{t}=O_{\mathbb{P}}(1)$ and since $\varepsilon_{t}$ and $X_{t}$ are independent, we have $n^{-1} \sum_{t=1}^{[n \pi]} \varepsilon_{t} X_{t}=o_{\mathbb{P}}(1)$. Thus, since $\psi^{\prime}$ is bounded, $\tilde{s}_{n}-\sigma_{0}=o_{\mathbb{P}}(1), \tilde{s}_{n}>0$ with probability 1 , the last term of the righthand side of (20) is $o_{\mathbb{P}}(1)$.

We now consider the second term of the right-hand side of (201). For the sum, we have:

$$
n^{-1}\left\|\sum_{t=1}^{[n \pi]} r_{t}\left(\tilde{\beta}_{1 n}\right) \psi\left(\frac{r_{t}\left(\tilde{\beta}_{1 n}\right)}{\tilde{s}_{n}}\right)-\sum_{t=1}^{[n \pi]} r_{t}\left(\beta_{1}^{0}\right) \psi\left(\frac{r_{t}\left(\beta_{1}^{0}\right)}{\sigma_{0}}\right)\right\|
$$


$\leq n^{-1}\left\|\sum_{t=1}^{[n \pi]}\left[r_{t}\left(\tilde{\beta}_{1 n}\right)-r_{t}\left(\beta_{1}^{0}\right)\right] \psi\left(\frac{r_{t}\left(\tilde{\beta}_{1 n}\right)}{\tilde{s}_{n}}\right)\right\|+n^{-1}\left\|\sum_{t=1}^{\| n \pi]} r_{t}\left(\beta_{1}^{0}\right)\left[\psi\left(\frac{r_{t}\left(\tilde{\beta}_{1 n}\right)}{\tilde{s}_{n}}\right)-\psi\left(\frac{r_{t}\left(\beta_{1}^{0}\right)}{\sigma_{0}}\right)\right]\right\|$

and since $\psi$ is bounded:

$$
\leq C\left\|\tilde{\beta}_{1 n}-\beta_{1}^{0}\right\| n^{-1} \sum_{t=1}^{[n \pi]}\left\|X_{t}\right\|+C n^{-1} \sum_{t=1}^{[n \pi]}\left\|\varepsilon_{t}\right\|
$$

The above inequality, with the ergodic theorem, $\mathbb{E}\left[\left\|X_{t}\right\|\right]<\infty, \mathbb{E}\left[\left\|\varepsilon_{t}\right\|\right]<\infty$ and $\tilde{\beta}_{1 n}-\beta_{1}^{0}=o_{\mathbb{P}}(1)$, imply that

$$
n^{-1} \sum_{t=1}^{[n \pi]} r_{t}\left(\tilde{\beta}_{1 n}\right) \psi\left(\frac{r_{t}\left(\tilde{\beta}_{1 n}\right)}{\tilde{s}_{n}}\right)-n^{-1} \sum_{t=1}^{[n \pi]} r_{t}\left(\beta_{1}^{0}\right) \psi\left(\frac{r_{t}\left(\beta_{1}^{0}\right)}{\sigma_{0}}\right)=o_{\mathbb{P}}(1)
$$

Thus, the second term of the right-hand side of (20) can be expressed:

$$
n^{-1} \sum_{t=1}^{[n \pi]} h_{t} \psi\left(x_{t}\right)=\frac{\tilde{s}_{n}-\sigma_{0}}{\sigma_{0} \tilde{s}_{n}}\left(n^{-1} \sum_{t=1}^{[n \pi]} \varepsilon_{t} \psi\left(\frac{\varepsilon_{t}}{\sigma_{0}}\right)+o_{\mathbb{P}}(1)\right)
$$

Then, relation (201) becomes:

$$
n^{-1} \sum_{t=1}^{[n \pi]} \chi\left(x_{t}+h_{t}\right)=n^{-1} \sum_{t=1}^{[n \pi]} \chi\left(x_{t}\right)+\frac{\tilde{s}_{n}-\sigma_{0}}{\sigma_{0} \tilde{s}_{n}}\left(n^{-1} \sum_{t=1}^{[n \pi]} \varepsilon_{t} \psi\left(\frac{\varepsilon_{t}}{\sigma_{0}}\right)+o_{\mathbb{P}}(1)\right)
$$

A similar relation holds for the part $t=[n \pi]+1, \cdots, n$ :

$$
n^{-1} \sum_{t=[n \pi]+1}^{n} \chi\left(x_{t}+h_{t}\right)=n^{-1} \sum_{t=[n \pi]+1}^{n} \chi\left(x_{t}\right)+\frac{\tilde{s}_{n}-\sigma_{0}}{\sigma_{0} \tilde{s}_{n}}\left(n^{-1} \sum_{t=[n \pi]+1}^{n} \varepsilon_{t} \psi\left(\frac{\varepsilon_{t}}{\sigma_{0}}\right)+o_{\mathbb{P}}(1)\right)
$$

Adding (21) and (22), taking into account the relation (19), we obtain:

$0=n^{-1} \sum_{t=1}^{[n \pi]} \chi\left(\frac{r_{t}\left(\tilde{\beta}_{1 n}\right)}{\sigma_{0}}\right)+n^{-1} \sum_{t=[n \pi]+1}^{n} \chi\left(\frac{r_{t}\left(\tilde{\beta}_{2 n}\right)}{\sigma_{0}}\right)+\frac{\tilde{s}_{n}-\sigma_{0}}{\sigma_{0} \tilde{s}_{n}}\left(n^{-1} \sum_{t=1}^{n} \varepsilon_{t} \psi\left(\frac{\varepsilon_{t}}{\sigma_{0}}\right)+o_{\mathbb{P}}(1)\right)$

By ergodic theorem: $n^{-1} \sum_{t=1}^{n} \varepsilon_{t} \psi\left(\varepsilon_{t} / \sigma_{0}\right) \underset{n \rightarrow \infty}{\stackrel{\mathbb{P}}{\longrightarrow}} \mathbb{E}\left[\varepsilon_{1} \psi\left(\varepsilon_{1} / \sigma_{0}\right)\right]$.

Stage 2. Then, the convergence rate of $\tilde{s}_{n}$ will be obtained by studying:

$n^{-1} \sum_{t=1}^{[n \pi]} \chi\left(\frac{r_{t}\left(\tilde{\beta}_{1 n}\right)}{\sigma_{0}}\right)+n^{-1} \sum_{t=[n \pi]+1}^{n} \chi\left(\frac{r_{t}\left(\tilde{\beta}_{2 n}\right)}{\sigma_{0}}\right)=\frac{\sigma_{0}-\tilde{s}_{n}}{\sigma_{0} \tilde{s}_{n}}\left[\mathbb{E}\left[\varepsilon_{1} \psi\left(\frac{\varepsilon_{1}}{\sigma_{0}}\right)\right]+o_{\mathbb{P}}(1)\right]$ 
For $t=1, \cdots,[n \pi]$, making the Taylor's expansion of $\chi$ up to second order, we obtain that $n^{-1} \sum_{t=1}^{[n \pi]} \chi\left(\sigma_{0}^{-1} r_{t}\left(\tilde{\beta}_{1 n}\right)\right)$ can be written as:

$$
n^{-1}\left\{\sum_{t=1}^{[n \pi]} \chi\left(\frac{\varepsilon_{t}}{\sigma_{0}}\right)-\frac{1}{\sigma_{0}} \sum_{t=1}^{[n \pi]} \chi^{\prime}\left(\frac{\varepsilon_{t}}{\sigma_{0}}\right) X_{t}\left(\tilde{\beta}_{1 n}-\beta_{1}^{0}\right)-\frac{1}{2 \sigma_{0}^{2}} \sum_{t=1}^{[n \pi]} \chi^{\prime \prime}\left(\frac{\varepsilon_{t}-\delta_{t} X_{t}\left(\tilde{\beta}_{1 n}-\beta_{1}^{0}\right)}{\sigma_{0}}\right)\left[X_{t}\left(\tilde{\beta}_{1 n}-\beta_{1}^{0}\right)\right]^{2}\right.
$$

Let us analyse the three terms of the previous equation separately.

- For the first term, let us $\nu_{t}=\varepsilon_{t} / \sigma_{0} \sim \mathcal{N}(0,1)$ denote. We use the Hermite expansion for $\sum_{t=1}^{[n \pi]} \chi\left(\nu_{t}\right)$. Because the Hermite rank of $\chi\left(\nu_{t}\right)$ is $q_{1}, q_{1} \geq 2$, by (13) below:

$$
\sum_{t=1}^{[n \pi]} \chi\left(\nu_{t}\right)=\frac{J_{q_{1}}(\chi)}{q_{1} !} \sum_{t=1}^{[n \pi]} H_{q_{1}}\left(\nu_{t}\right)+\sum_{t=1}^{[n \pi]} \sum_{q \geq q_{1}+1} \frac{J_{q}(\chi)}{q !} H_{q}\left(\nu_{t}\right): \equiv T_{1, n}+T_{2, n}
$$

For $T_{1, n}$ we have:

$$
\begin{gathered}
\mathbb{E}\left[T_{1, n}^{2}\right]=\frac{J_{q_{1}}^{2}(\chi)}{\left(q_{1} !\right)^{2}} \sum_{t=1}^{[n \pi]} \sum_{j=1}^{[n \pi]}\left(q_{1} !\right) \gamma^{q 1}(|t-j|)=\left(q_{1} !\right) \frac{J_{1}^{2}(\rho)}{\left(q_{1} !\right)^{2}}\left[[n \pi] \gamma^{q_{1}}(0)+2 \sum_{t=1}^{[n \pi]-1}([n \pi]-t) \gamma^{q_{1}}(t)\right] \\
=\left(q_{1} !\right) \frac{J_{1}^{2}(\rho)}{\left(q_{1} !\right)^{2}}\left[O(n)+2 \sum_{t=1}^{[n \pi]-1}([n \pi]-t) t^{-\alpha q_{1}} L^{q_{1}}(t)\right]=\frac{J_{1}^{2}(\rho)}{q_{1} !}\left[O(n)+O\left(n^{2-\alpha q_{1}}\right) L^{q_{1}}([n \pi])\right] \\
=O\left(n^{2-\alpha q_{1}}\right) L^{q_{1}}([n \pi])
\end{gathered}
$$

For $T_{2, n}$ we have:

$$
\begin{gathered}
\mathbb{E}\left[T_{2, n}^{2}\right]=\sum_{q \geq q_{1}+1} \frac{J_{q}^{2}(\rho)}{q !}\left[\sum_{t=1}^{[n \pi]} \sum_{j=1}^{[n \pi]} \gamma^{q}(|t-j|)\right] \leq \sum_{q \geq q_{1}+1} \frac{J_{q}^{2}(\rho)}{q !} \sum_{t=1}^{[n \pi]} \sum_{j=1}^{[n \pi]} \gamma^{q_{1}+1}(|t-j|) \\
=O(n)+2 \sum_{q \geq q_{1}+1} \frac{J_{q}^{2}(\rho)}{q !} \sum_{t=1}^{[n \pi]-1}([n \pi]-t) \gamma^{q_{1}+1}(t) \leq O(n)+2 \sum_{q \geq q_{1}+1} \frac{J_{q}^{2}(\rho)}{q !} \sum_{t=1}^{[n \pi]-1}([n \pi]-t) t^{-\left(q_{1}+1\right) \alpha} L^{q_{1}+1}(t) \\
=O\left(n^{2-\left(q_{1}+1\right) \alpha} L^{q_{1}+1}([n \pi])\right)
\end{gathered}
$$

Hence $\mathbb{E}\left[T_{2, n}^{2}\right]=o\left(\mathbb{E}\left[T_{1, n}^{2}\right]\right)$. Then, for equation (25), we straightforwardly have:

$$
\sum_{t=1}^{[n \pi]} \chi\left(\nu_{t}\right)=O_{\mathbb{P}}\left(\mathbb{E}\left[T_{1, n}^{2}\right]\right)^{1 / 2}=O_{\mathbb{P}}\left(n^{1-\alpha q_{1} / 2}\right) L^{q_{1} / 2}([n \pi])
$$

- For the second term of (24), since $\nu_{t}$ and $X_{t}$ are independent, by ergodic theorem, 
we have:

$$
n^{-1} \sum_{t=1}^{[n \pi]} \chi^{\prime}\left(\nu_{t}\right) X_{t}\left(\tilde{\beta}_{1 n}-\beta_{1}^{0}\right)=o_{\mathbb{P}}\left(\left\|\tilde{\beta}_{1 n}-\beta_{1}^{0}\right\|\right)
$$

- For the third term of (24), since $\psi^{\prime}$ is bounded and $n^{-1} \sum_{t=1}^{[n \pi]} X_{t} X_{t}^{T}=O_{\mathbb{P}}(1)$, we have:

$$
n^{-1} \sum_{t=1}^{[n \pi]} \chi^{\prime \prime}\left(\nu_{t}\right)\left[X_{t}\left(\tilde{\beta}_{1 n}-\beta_{1}^{0}\right)\right]^{2}=O_{\mathbb{P}}\left(\left\|\tilde{\beta}_{1 n}-\beta_{1}^{0}\right\|^{2}\right)=o_{\mathbb{P}}\left(\left\|\tilde{\beta}_{1 n}-\beta_{1}^{0}\right\|\right)
$$

Then, by taking (26), (27), (28) into account, the behaviour of (24) is given by (26) and it is $O_{\mathbb{P}}\left(n^{-\alpha q_{1} / 2}\right) L^{q_{1} / 2}([n \pi])+o_{\mathbb{P}}\left(\left\|\tilde{\beta}_{1 n}-\beta_{1}^{0}\right\|\right)$. Similar one reasoning is made for the part $t=[n \pi]+1, \cdots, n$ and we obtain that: $n^{-1} \sum_{t=[n \pi]+1}^{n} \chi\left(\sigma_{0}^{-1} r_{t}\left(\tilde{\beta}_{2 n}\right)\right)=$ $O_{\mathbb{P}}\left(n^{-\alpha q_{1} / 2}\right) L^{q_{1} / 2}(n(1-[\pi]))+o_{\mathbb{P}}\left(\left\|\tilde{\beta}_{2 n}-\beta_{2}^{0}\right\|\right)$. Then, for relation (23) , we have:

$\frac{\sigma_{0}-\tilde{s}_{n}}{\sigma_{0} \tilde{s}_{n}}\left[\mathbb{E}\left[\varepsilon_{1} \psi\left(\frac{\varepsilon_{1}}{\sigma_{0}}\right)\right]+o_{\mathbb{P}}(1)\right]=O_{\mathbb{P}}\left(n^{-\alpha q_{1} / 2}\right) L^{q_{1} / 2}(n)+o_{\mathbb{P}}\left(\left\|\tilde{\beta}_{1 n}-\beta_{1}^{0}\right\|+\left\|\tilde{\beta}_{2 n}-\beta_{2}^{0}\right\|\right)$ and the convergence rate of $\tilde{s}_{n}$ follows.

Proof of Theorem 3.4. As a consequence of Theorem 3.1, we consider $\pi$ in a neighbourhood of $\pi^{0}$. We suppose, without loss of generality, that $\pi<\pi^{0}$. Considering relation (12) (c), we have:

$$
n^{-1} \sum_{t=[n \pi]+1}^{\left[n \pi^{0}\right]} \psi\left(\frac{r_{t}\left(\tilde{\beta}_{2 n}(\pi)\right)}{s_{n}\left(\tilde{\beta}_{1 n}(\pi), \tilde{\beta}_{2 n}(\pi), \pi\right)}\right) X_{t}=-n^{-1} \sum_{t=\left[n \pi^{0}\right]+1}^{n} \psi\left(\frac{r_{t}\left(\tilde{\beta}_{2 n}(\pi)\right)}{s_{n}\left(\tilde{\beta}_{1 n}(\pi), \tilde{\beta}_{2 n}(\pi), \pi\right)}\right) X_{t}
$$

Since $\left\|\tilde{\beta}_{2 n}(\pi)-\beta_{2}^{0}\right\|=O_{\mathbb{P}}\left(n^{-k} \tilde{L}_{1}(n)\right)$, an argument like the one used for relation (27) yield that the right-hand side of (29) is $O_{\mathbb{P}}\left(n^{-k} \tilde{L}_{1}(n)\right)$.

We apply (8) to function $\psi$, for: $x_{t}=\frac{\varepsilon_{t}}{s_{n}\left(\tilde{\beta}_{1 n}(\pi), \tilde{\beta}_{2 n}(\pi), \pi\right)}, h_{t}=-\frac{X_{t}\left(\tilde{\beta}_{2 n}(\pi)-\beta_{1}^{0}\right)}{s_{n}\left(\tilde{\beta}_{1 n}(\pi), \tilde{\beta}_{2 n}(\pi), \pi\right)}$. For the left-hand side of (29) , since $s_{n}\left(\tilde{\beta}_{1 n}(\pi), \tilde{\beta}_{2 n}(\pi), \pi\right) \rightarrow \sigma_{0}$ a.s. for $n \rightarrow \infty$, and $\beta_{1}^{0} \neq \beta_{2}^{0}$, we obtain :

$$
n^{-1} \sum_{t=[n \pi]+1}^{\left[n \pi^{0}\right]} \psi\left(\frac{r_{t}\left(\tilde{\beta}_{2 n}(\pi)\right)}{s_{n}\left(\tilde{\beta}_{1 n}(\pi), \tilde{\beta}_{2 n}(\pi), \pi\right)}\right) X_{t}=n^{-1} \sum_{t=[n \pi]+1}^{\left[n \pi^{0}\right]} \psi\left(\frac{\varepsilon_{t}}{\sigma_{0}}\right) X_{t}+O_{\mathbb{P}}\left(n\left(\pi^{0}-\pi\right)\right)
$$

But, making Hermite expansion of $\psi\left(\nu_{t}\right)$, we get:

$$
\sum_{t=[n \pi]+1}^{\left[n \pi^{0}\right]} \psi\left(\frac{\varepsilon_{t}}{\sigma_{0}}\right) X_{t}=\frac{J_{1}(\psi)}{\sigma_{0}} \sum_{t=[n \pi]+1}^{\left[n \pi^{0}\right]} \varepsilon_{t} X_{t}+\sum_{t=[n \pi]+1}^{\left[n \pi^{0}\right]} \sum_{q>1} \frac{J_{q}(\psi)}{q !} H_{q}\left(\nu_{t}\right) X_{t}: \equiv I_{1, n}+I_{2, n}
$$


where: $J_{q}(\psi)=\mathbb{E}\left[\psi\left(\nu_{1}\right) H_{q}\left(\nu_{1}\right)\right]$. On the other hand, as in the proof of Theorem 3.3, we have $I_{2, n}=o_{\mathbb{P}}\left(I_{1, n}\right)$. The variance of $I_{1, n}$ is:

$$
\begin{aligned}
& \mathbb{E}\left[I_{1, n} I_{1, n}^{T}\right]=\frac{J_{1}^{2}(\psi)}{\sigma_{0}^{2}} \sum_{i=1}^{\left[n\left(\pi^{0}-\pi\right)\right]} \sum_{j=1}^{\left[n\left(\pi^{0}-\pi\right)\right]} \gamma(|i-j|) \Gamma(|i-j|) \\
= & {\left[n\left(\pi^{0}-\pi\right)\right] \gamma(0) \Gamma(0)+2 \sum_{i=1}^{\left[n\left(\pi^{0}-\pi\right)\right]}\left[n\left(\pi^{0}-\pi\right)-i\right] \gamma(i) \Gamma(i) } \\
= & O\left(L\left(n\left(\pi^{0}-\pi\right)\right) \mathcal{L}^{T}\left(n\left(\pi^{0}-\pi\right)\right) M\left(n\left(\pi^{0}-\pi\right)\right) \mathcal{L}\left(n\left(\pi^{0}-\pi\right)\right)\right)
\end{aligned}
$$

What implies:

$n^{-1} \sum_{t=[n \pi]+1}^{\left[n \pi^{0}\right]} \psi\left(\frac{\varepsilon_{t}}{\sigma_{0}}\right) X_{t}=O_{\mathbb{P}}\left(\left(n\left(\pi^{0}-\pi\right)\right)^{-\min \left(\theta_{i}+\alpha\right) / 2} L^{1 / 2}\left(n\left(\pi^{0}-\pi\right)\right) \mathcal{L}^{T}\left(n\left(\pi^{0}-\pi\right)\right) \mathcal{L}\left(n\left(\pi^{0}-\pi\right)\right)\right)$

This last relation together with (29), (30) and since the right-hand side of (29) is $O_{\mathbb{P}}\left(n^{-k} \tilde{L}_{1}(n)\right)$ imply: $O_{\mathbb{P}}\left(n^{-k} \tilde{L}_{1}(n)\right)$

$=O_{\mathbb{P}}\left(n\left(\pi^{0}-\pi\right)\right)+O_{\mathbb{P}}\left(\left(n\left(\pi^{0}-\pi\right)\right)^{-\min \left(\theta_{i}+\alpha\right) / 2} L^{1 / 2}\left(n\left(\pi^{0}-\pi\right)\right) \mathcal{L}^{T}\left(n\left(\pi^{0}-\pi\right)\right) \mathcal{L}\left(n\left(\pi^{0}-\pi\right)\right)\right)$

We obtain that: $\hat{\pi}_{n}-\pi^{0}=O_{\mathbb{P}}\left(n^{-1-k} \tilde{L}_{1}(n)\right)$.

\section{Lemmas}

Lemma 5.1 If solution $s_{n}(\xi)$ of equation (4) exists, then it is well-defined, bounded, strictly positive, with a probability arbitrarily large.

Proof of Lemma 5.1. Since $\mathbb{E}\left[r_{t}(\beta)=0\right]$ and $\operatorname{Var}\left[r_{t}(\beta)\right]=\operatorname{Var}\left[\varepsilon_{t}\right]+\beta \operatorname{Var}[X] \beta^{t}<$ $\infty$, by Bienaymé-Tchebichev inequality, we obtain that $r_{t}(\beta)$ is bounded with a probability arbitrarily large.

We prove that $s_{n}(\xi)$ is bounded by reduction to absurdity. If $s_{n}(\xi)$ is not bounded then: there exists $\xi \in \Upsilon \times \Upsilon \times(0,1)$ and $n_{\xi} \in \mathbb{N}$ such that for all $n>n_{\xi}, M>0$, exists $\epsilon>0$ such that: $\mathbb{P}\left[s_{n}(\xi)>M\right] \geq 1-\epsilon$. Since $\rho$ is continuous and $\rho(0)=0$, then:

$$
\rho\left(\frac{r_{t}(\beta)}{s_{n}(\xi)}\right) \underset{n \rightarrow \infty}{\stackrel{\mathbb{P}}{\longrightarrow}} 0, \quad t=1, \ldots, n
$$


and

$$
\begin{gathered}
\frac{1}{n} \sum_{t=1}^{[n \pi]} \rho\left(\frac{r_{t}\left(\beta_{1}\right)}{s_{n}(\xi)}\right)+\frac{1}{n} \sum_{t=[n \pi]+1}^{n} \rho\left(\frac{r_{t}\left(\beta_{2}\right)}{s_{n}(\xi)}\right) \\
\leq \frac{[n \pi]}{n} \max _{1 \leq t \leq[n \pi]} \rho\left(\frac{r_{t}\left(\beta_{1}\right)}{s_{n}(\xi)}\right)+\frac{n-[n \pi]}{n} \max _{[n \pi]+1 \leq t \leq n} \rho\left(\frac{r_{t}\left(\beta_{2}\right)}{s_{n}(\xi)}\right)
\end{gathered}
$$

which, by (31), converges to 0 in probability, for $n \rightarrow \infty$. What is contradictory with (44). To prove that $s_{n}(\xi)>0$, let us consider function $g(\beta, s)=(\varepsilon-X \beta) / s$, with $\beta$ in a compact of $\mathbb{R}^{d}$ containing 0 and $s \in(0, \infty)$. Since $\varepsilon-X \beta$ is bounded with a probability close to 1 , if $s_{n}(\xi)=0$, thus $\lim _{s \rightarrow 0}|g(\beta, s)|=\infty$, what is contradictory with (44). Hence, for all $\epsilon>0$, there exists $\delta>0$ such that $\mathbb{P}\left[\inf _{\xi \in \Upsilon \times \Upsilon \times[0,1]} s_{n}(\xi)>\delta\right]>1-\epsilon$.

Lemma 5.2 Under assumptions (A1)-(A3), for any $\epsilon \in(0,1), \xi \in \Upsilon \times \Upsilon \times[0,1]$, there exists a positive constant $\delta$ such that: $\mathbb{P}\left[\inf _{\xi \in \Upsilon \times \Upsilon \times[0,1]} D_{n}(\xi)>\delta\right]>1-\epsilon$.

Proof of Lemma [5.2. Because $\xi$ belongs to a compact and taking into account relation (11), we have to prove that for all $\epsilon>0, \xi \in \Upsilon \times \Upsilon \times[0,1]$, there exists a $\delta>0$ such that:

$$
\mathbb{P}\left[n^{-1}\left[\sum_{t=1}^{[n \pi]} r_{t}\left(\beta_{1}\right) \psi\left(\frac{r_{t}\left(\beta_{1}\right)}{s_{n}(\xi)}\right)+\sum_{t=[n \pi]+1}^{n} r_{t}\left(\beta_{2}\right) \psi\left(\frac{r_{t}\left(\beta_{2}\right)}{s_{n}(\xi)}\right)\right]>\delta\right]>1-\epsilon
$$

Since $r_{t}(\beta), \psi\left(\frac{r_{t}(\beta)}{s_{n}(\xi)}\right)$ have the same sign and since $\psi$ is continuous, we are going to show only that, for all $\epsilon>0$, for all $\beta$ in compact set $\Upsilon$, there exists a $\delta_{1}>0$ such that: $\mathbb{P}\left[|\varepsilon-X \beta|>\delta_{1}\right]>1-\epsilon$.

Random variables $\varepsilon$ and $X$ are Gaussian and independent. Then: $\mathbb{P}[|\varepsilon-X \beta|>$ $\left.\delta_{1}\right]=2 \mathbb{P}\left[\varepsilon-X \beta<-\delta_{1}\right]=2 \Phi\left(-\frac{\delta_{1}}{\left[\gamma(0)+\beta \Gamma(0) \beta^{T}\right]^{1 / 2}}\right)$. We recall that $\Phi$ denotes the standard Gaussian distribution. Then, the Lemma results by setting: $\delta_{1}=$ $\inf _{\beta \in \Upsilon}\left[\gamma(0)+\beta \Gamma(0) \beta^{T}\right]^{1 / 2}\left|\Phi^{-1}\left(\frac{1-\epsilon}{2}\right)\right|$.

The key for strong convergence proof is the following uniform convergence result.

Lemma 5.3 For all $\varrho>0$, under assumptions (A1)-(A3), for $\Omega_{\varrho}(\xi)=\left\{\xi^{*} \in \Upsilon \times \Upsilon \times[0,1] ;\left\|\eta-\eta^{*}\right\|<\varrho,\left|\pi-\pi^{*}\right|<\varrho\right\}$, we have:

$$
\mathbb{E}\left[\sup _{\xi^{*} \in \Omega_{\varrho}(\xi)}\left|s_{n}(\eta, \pi)-s_{n}\left(\eta^{*}, \pi^{*}\right)\right|\right] \underset{\varrho \rightarrow 0}{\longrightarrow} 0
$$


Proof of Lemma 5.3. We have the triangular inequality:

$\left|s_{n}(\eta, \pi)-s_{n}\left(\eta^{*}, \pi^{*}\right)\right| \leq\left|s_{n}(\eta, \pi)-s_{n}\left(\eta, \pi^{*}\right)\right|+\left|s_{n}\left(\eta, \pi^{*}\right)-s_{n}\left(\eta^{*}, \pi^{*}\right)\right|$. First, we will study $s_{n}\left(\eta, \pi^{*}\right)-s_{n}\left(\eta^{*}, \pi^{*}\right)$. By the mean value theorem (TVM), we have:

$$
s_{n}\left(\eta, \pi^{*}\right)-s_{n}\left(\eta^{*}, \pi^{*}\right)=\left(\beta_{1}-\beta_{1}^{*}\right) \frac{\partial s_{n}}{\partial \beta_{1}}\left(\tilde{\beta}_{1}, \beta_{2}^{*}, \pi^{*}\right)+\left(\beta_{2}-\beta_{2}^{*}\right) \frac{\partial s_{n}}{\partial \beta_{2}}\left(\beta_{1}^{*}, \tilde{\beta}_{2}, \pi^{*}\right)
$$

where $\tilde{\beta}_{1}=\beta_{1}+v_{1}\left(\beta_{1}-\beta_{1}^{*}\right), \tilde{\beta}_{2}=\beta_{2}+v_{2}\left(\beta_{2}-\beta_{2}^{*}\right), v_{1}, v_{2} \in(0,1)$. By Lemma 5.2, applying Cauchy-Schwarz inequality in (10) and taking into account that $\psi$ is bounded, we obtain:

$$
\mathbb{E}\left[\left|\frac{\partial s_{n}\left(\tilde{\beta}_{1}, \beta_{2}^{*}, \pi^{*}\right)}{\partial \beta_{1}}\right|\right] \leq C n^{-1} \sum_{t=1}^{[n \pi]}\left(\mathbb{E}\left[X_{t}^{2}\right] \mathbb{E}\left[\psi^{2}\left(\frac{r_{t}\left(\tilde{\beta}_{1}\right)}{s_{n}\left(\tilde{\beta}_{1}, \beta_{2}^{*}, \pi^{*}\right)}\right)\right]\right)^{1 / 2}<C
$$

Then, writing a similar relation for $\left(\partial s_{n} / \partial \beta_{2}\right)\left(\beta_{1}^{*}, \tilde{\beta}_{2}, \pi^{*}\right)$, we have for (32):

$$
\mathbb{E}\left[\left|s_{n}\left(\eta, \pi^{*}\right)-s_{n}\left(\eta^{*}, \pi^{*}\right)\right|\right] \longrightarrow 0, \quad \text { for } \varrho \rightarrow 0
$$

Let us remark that if $\pi^{*}=0$ or $\pi^{*}=1$, then in relation (32), the term in $\beta_{1}$, respectively $\beta_{2}$, does not appear.

Now, we study $\left|s_{n}(\eta, \pi)-s_{n}\left(\eta, \pi^{*}\right)\right|$, supposing that $\pi<\pi^{*}$. Since $s_{n}(\eta, \pi)$ and $s_{n}\left(\eta, \pi^{*}\right)$ are both solutions of (4), we have:

$$
\begin{gathered}
n^{-1} \sum_{t=1}^{\left[n \pi^{*}\right]}\left[\rho\left(\frac{r_{t}\left(\beta_{1}\right)}{s_{n}(\eta, \pi)}\right)-\rho\left(\frac{r_{t}\left(\beta_{1}\right)}{s_{n}\left(\eta, \pi^{*}\right)}\right)\right]+n^{-1} \sum_{t=\left[n \pi^{*}\right]+1}^{n}\left[\rho\left(\frac{r_{t}\left(\beta_{2}\right)}{s_{n}(\eta, \pi)}\right)-\rho\left(\frac{r_{t}\left(\beta_{2}\right)}{s_{n}\left(\eta, \pi^{*}\right)}\right)\right] \\
=n^{-1} \sum_{[n \pi]+1}^{\left[n \pi^{*}\right]}\left[\rho\left(\frac{r_{t}\left(\beta_{1}\right)}{s_{n}(\eta, \pi)}\right)-\rho\left(\frac{r_{t}\left(\beta_{2}\right)}{s_{n}(\eta, \pi)}\right)\right]
\end{gathered}
$$

Thus, applying the MVT:

$$
\begin{aligned}
& n^{-1}\left[s_{n}\left(\eta, \pi^{*}\right)-s_{n}(\eta, \pi)\right]\left[\sum_{t=1}^{\left[n \pi^{*}\right]} r_{t}\left(\beta_{1}\right) \psi\left(\frac{r_{t}\left(\beta_{1}\right)}{u_{n}^{(1)}\left(\eta, \pi, \pi^{*}\right)}\right)+\sum_{t=\left[n \pi^{*}\right]+1}^{n} r_{t}\left(\beta_{2}\right) \psi\left(\frac{r_{t}\left(\beta_{2}\right)}{u_{n}^{(2)}\left(\eta, \pi, \pi^{*}\right)}\right)\right] \\
& =n^{-1} \sum_{[n \pi]+1}^{\left[n \pi^{*}\right]}\left[X_{t}\left(\beta_{1}-\beta_{2}\right)\right] \psi\left(\frac{\tilde{r}_{t}\left(\beta_{1}, \beta_{2}\right)}{s_{n}(\eta, \pi)}\right)
\end{aligned}
$$

where $u^{(1)}, u^{(2)}$ are two positive bounded functions, not necessarily solutions of (4) and $\tilde{r}_{t}\left(\beta_{1}, \beta_{2}\right)=r_{t}\left(\beta_{1}\right)+m_{t}\left[r_{t}\left(\beta_{2}\right)-r_{t}\left(\beta_{1}\right)\right]$, with $0<m_{t}<1$. By relation (11):

$$
\sum_{t=1}^{\left[n \pi^{*}\right]} r_{t}\left(\beta_{1}\right) \psi\left(\frac{r_{t}\left(\beta_{1}\right)}{u_{n}^{(1)}\left(\eta, \pi, \pi^{*}\right)}\right)+\sum_{t=\left[n \pi^{*}\right]+1}^{n} r_{t}\left(\beta_{2}\right) \psi\left(\frac{r_{t}\left(\beta_{2}\right)}{u_{n}^{(2)}\left(\eta, \pi, \pi^{*}\right)}\right)>0
$$


with a probability close to 1 . On the other hand: $\tilde{r}_{t}\left(\beta_{1}, \beta_{2}\right)=Y_{t}-X_{t}\left[\beta_{1}+m_{t}\left(\beta_{2}-\right.\right.$ $\left.\left.\beta_{1}\right)\right]=r_{t}\left(\beta_{1}+m_{t}\left(\beta_{2}-\beta_{1}\right)\right)$. Using the same arguments as for (33), we obtain that:

$$
n^{-1} \sum_{t=[n \pi]+1}^{\left[n \pi^{*}\right]}\left(\mathbb{E}\left[X_{t}^{2}\right] \mathbb{E}\left[\psi^{2}\left(\frac{\tilde{r}_{t}\left(\beta_{1}, \beta_{2}\right)}{s_{n}(\eta, \pi)}\right)\right]\right)^{1 / 2} \leq C_{1}\left(\pi^{*}-\pi\right)
$$

where $C_{1}$ is a vector with all bounded components. Taking into account also (36), we obtain for (35):

$$
\mathbb{E}\left[\left|s_{n}(\eta, \pi)-s_{n}\left(\eta, \pi^{*}\right)\right|\right] \leq C\left\|\beta_{1}-\beta_{2}\right\| \cdot\left|\pi-\pi^{*}\right|<C \varrho \underset{\varrho \rightarrow 0}{\longrightarrow} 0
$$

Relations (34) and (37) imply the Lemma.

\section{References}

[1] J. Bai, Least squares estimation of a shift in linear processes, Journal of Time Series Analysis 15 (1994) 453-472.

[2] J. Bai, Estimation of multiple-regime regressions with least absolute deviation, Journal of Statistical Planning Inference, 74 (1998) 103-134.

[3] J. Bai, P. Perron, Estimating and testing linear models with multiple structural changes, Econometrica 66 (1998) 47-78.

[4] R. Baillie, Long memory processes and fractional integration in econometrics, Journal of Econometrics, 73 (1996) 5-59.

[5] A.E. Beaton, J.W. Tukey, The fitting of power series, meaning polynomials, illustrated on band-spectroscopic data, Technometrics 16 (1974) 147-185.

[6] J. Beran, Statistics for long-memory process, Chapman \& Hall, New York, 1994.

[7] P.K.Bhattacharya, Some aspects of change-point analysis. IMS Lecture NotesMonograph Series, Vol. 23, Hayward, CA 1994, pp.28-56.

[8] Y.X. Cheung, Long memory in foreign exchange rates, Journal of Bussiness and Economic Statistics 11(1993) 93-101.

[9] G. Ciuperca, N. Dapzol, Maximum likelihood estimator in a multi-phase random regression model, Statistics 42 (2008) 363-381.

[10] G. Ciuperca Estimating nonlinear regression with and without change-points by the LAD-method, Annals of the Institute of Statistical Mathematics in revision. 
[11] L. Davies, The Asymptotics of S-Estimators in the Linear Regression Model, Annals of Statistics 18 (1990) 1651-1675.

[12] Z. Ding, C.W.J. Granger, R.F. Engle, A long memory property of stock market returns and a new model, Journal of Empirical Finance 1 (1993) 83-106.

[13] P.I. Feder, On asymptotic distribution theory in segmented regression problemsidentified case, Ann. Statist. 3 (1975) 49-83.

[14] P.I. Feder, The log likelihood ratio in segmented regression, Ann. Statist. 3 (1975) 84-97.

[15] I. Fiteni, Robust estimation of structural break points, Econometric Theory 18 (2002) 349-386.

[16] I. Fiteni, $\tau$-estimators of regression models with structural change of unknown location, Journal of Econometrics 119 (2004) 19-44.

[17] H. Guo, H.L. Koul, Nonparametric regression with heteroscedastic long memory errors, Journal of Statistical Planning Inference 137 (2007) 379-404.

[18] F.R.. Hampel, A general quantitative definition of robustness, Annals of Mathematical Statistics 42 (1971) 1887-1896.

[19] J. Hidalgo, P.M. Robinson, Testing for structural change in a long-memory environment, Journal of Econometrics 1 (1996) 159-174.

[20] L. Horvath, P. Kokoszka, The effect of long-range dependence on change-point estimators, Journal of Statistical Planning Inference 64 (1997) 57-81.

[21] P.J. Huber, The behaviour of maximum likelihood estimates under nonstandard conditions. Proceedings of the Fifth Berkeley Symposium on Mathematics Statistic and Probability, Vol 1, University California Press, Berkeley, 1967, pp 221-234.

[22] J. Kim , H.J Kim, Asymptotic results in segmented multiple regression, Journal of Multivariate Analysis 99 (2008) 2016-2038.

[23] H.L. Koul, L. Qian, Asymptotics of maximum likelihood estimator in a two-phase linear regression model, Journal of Statistical Planning and Inference 108 (2002) 99-119.

[24] H.L. Koul, R.T. Baillie, Asymptotics of M-estimators in non-linear regression with long memory design, Statistics and Probability Letters 61 (2003) 237-252.

[25] H.L. Koul, L. Qian, D. Surgailis, Asymptotics of M-estimators in two-phase linear regression models, Stochastic Processes and their Applications 103 (2003) 123-154.

[26] C.M. Kuan, C.C. Hsu, Change-point estimation of fractionally integrated processes, Journal of Time Series Analysis 19 (1998) 693-708. 
[27] S. Lazarová, Testing for structural change in regression with long memory processes, Journal of Econometrics 129 (2005) 329-372.

[28] A.W. Lo, Long term memory in stock market prices, Econometrica 59 (1991) 1279-1313.

[29] L.C. Nunes, C.M. Kuan, P. Newbold, Spurious break Econometric Theory 11(1995) 736-749.

[30] W. Palma, Long-Memory Time Series, Theory and Methods, Wiley, New Jersey, 2007.

[31] P.M. Robinson, Time series with strong dependence, In: C.A. Sims, Advances in Econometrics, Sixth World Congress, Cambridge Univ. Press, 1994.

[32] E. Roelant, S. Van Aelst, C. Croux, Multivariate generalized S-estimators Journal of Multivariate Analysis (2008) in press.

[33] P.J. Rousseeuw, V.J. Yohai, Robust regression by means of S-estimators, In: Robust and Nonlinear Time Series Analysis, Franke, J., Hrdle, W., Martin, R.D. (Eds.), Lecture Notes in Statistics, Vol. 26, Springer, New York, 1984, pp. 256-272.

[34] A.L. Rukhin, I. Vajda, Change-point estimation as a nonlinear regression problem, Statistics 30 (1997) 181-200.

[35] Ph. Sibbertsen, Long memory versus structural breaks: an overview, Statistical Paper 4 (2004) 465-515.

[36] L. Zhengyan,L. Degui, C. Jia, Asymptotic behavior for $S$-estimators in random design linear model with long-range-dependent errors, Metrika 66 (2007) 289-303. 